\title{
SUCESSÃO VEGETAL EM UMA ENCOSTA REFLORESTADA COM LEGUMINOSAS ARBÓREAS EM ANGRA DOS REIS, RJ'
}

\author{
Sylvia de Souza Chada ${ }^{2}$, Eduardo Francia Carneiro Campello ${ }^{3}$ e Sérgio Miana de Faria ${ }^{4}$
}

\begin{abstract}
Resumo - Em uma encosta reflorestada há sete anos com leguminosas arbóreas (Acacia auriculiformis, A. mangium e Mimosa tenuiflora) em Angra dos Reis, RJ, foi avaliada a composição florística e fitossociológica da regeneração natural, comparando-as com as de um fragmento de Mata Secundária situado a 200 m de distância. Foram considerados os três terços da encosta, com declividades decrescentes. Em 12 parcelas de $200 \mathrm{~m}^{2}$, quatro em cada terço da encosta, foram amostrados 699 indivíduos vegetais a partir de $40 \mathrm{~cm}$ de altura, distribuídos em 25 famílias e 50 espécies. As famílias com maior $n^{\circ}$ de indivíduos foram Meliaceae (298), Euphorbiaceae (70), Piperaceae (64) e Lauraceae (41). Já as famílias com maior $n^{\circ}$ de espécies foram Solanaceae (7), Melastomataceae (5) e Myrtaceae (5). As leguminosas plantadas não estavam regenerando na própria área. A evolução da sucessão natural apresentou um gradiente de desenvolvimento em razão da menor declividade e menor distância dos remanescentes florestais, com maior densidade de indivíduos e maior riqueza de espécies na área de menor declividade.
\end{abstract}

Palavras-chave: sucessão, leguminosas florestais e reabilitação florestal.

\section{NATURAL SUCCESSION UNDER A NITROGEN-FIXING LEGUME TREES STAND IN A HILLSIDE AT ANGRA DOS REIS - RJ, BRAZIL}

\begin{abstract}
The floristic composition and natural regeneration under a 7-year-old le gume tree plantation (Acacia auriculiformis, A. mangium e Mimosa tenuiflora) was investigated in comparing with a secondary forest $200 \mathrm{~m}$ away at Angra dos Reis, RJ. The hillside was divided in 3 parts following the slope. The lower part of the hillside was the nearest to the natural forest remnant. In 12 plots with $200 \mathrm{~m}^{2}$ each, 4 of them in each section of the hillside, 699 plants larger then $40 \mathrm{~cm}$ height were observed, distributed in 25 families and 50 species. The families with the most individuals were Meliaceae (298), Euphorbiaceae (70), Piperaceae (64) and Lauraceae (41). The families with the most species were Solanaceae (7), Melastomataceae (5) and Myrtaceae (5). None of the legume species introduced in the area had produced natural regeneration. The evolution of natural succession acurred with a gradient in function of the slope and distance of the remnant natural forest. The higher density of individuals and species richness were found in the lowest part of the hillside.
\end{abstract}

Key words: Succession, legume trees, forest rehabilitation

\section{INTRODUÇÃO}

A reabilitação de ecossistemas florestais pode ser alcançada através do plantio de espécies facilitadoras da sucessão natural, em locais onde, a princípio, uma série de barreiras impede o desenvolvimento do processo. A capacidade de estabelecimento em condições limitantes, a atração de fauna, o crescimento rápido e a grande deposição de serrapilheira são características desejáveis de espécies para plantios de reabilitação.

Espécies da família Leguminosae que estabelecem simbiose eficiente com bactérias fixadoras de $\mathrm{N}_{2}$ atmosférico apresentam uma vantagem adicional para

\footnotetext{
${ }^{1}$ Recebido em 14.12.2002 e aceito para publicação em 10.08.2004.

${ }^{2}$ Enga . Agrônoma. E-mail: <sylviachada@bol.com.br>.

${ }^{3}$ Embrapa Agrobiologia. E-mail: <campello@ cnpab.embrapa.br >.

${ }^{4}$ Embrapa Agrobiologia. E-mail: <sdefaria@ cnpab.embrapa.br>.
} 
plantios de reabilitação, considerando-se que em condições tropicais o nitrogênio é, em geral, extremamente limitante (FRANCO et al., 1992; FRANCO et al., 1995; FRANCO e FARIA, 1997). Entretanto, o uso de espécies de uma única família botânica é questionado por alguns autores (REIS et al., 1996; KAGEYAMA et al., 1994), que consideram importante ter a diversidade original do ecossistema como modelo, para não se correr o risco de inibição do processo de sucessão que completaria o de recuperação.

Estudos da regeneração natural sob plantios monoespecíficos de eucalipto, por exemplo (SILVA JR. et al., 1995; CALEGÁRIO, 1992), indicaram que esta espécie não reduziu a diversidade de espécies em seu sub-bosque. Haggar et al. (1997) observaram que árvores de estabelecimento rápido, com altas taxas de crescimento, no geral estimulam níveis mais altos de regeneração em seu sub-bosque. Campello (1999), realizando levantamento florístico da regeneração natural sob plantios monoespecíficos de leguminosas, nativas e exóticas, e não-leguminosas, também nativas e exóticas, concluiu que a regeneração natural das espécies nativas foi beneficiada pelos plantios de leguminosas arbóreas, independentemente da origem das espécies plantadas.

O processo de reabilitação de áreas degradadas está condicionado também à oferta de propágulos, em função da distância e da qualidade da fonte e dos agentes dispersores. A distância dos remanescentes florestais em relação à área em processo de reabilitação vai influenciar a quantidade de propágulos que chega à área (PARROTA, 1993), assim como a qualidade da fonte, entendida aqui como a riqueza de espécies da vegetação circunvizinha. Em sistemas implantados, é de fundamental importância o estabelecimento de indicadores de avaliação e monitoramento. Aregeneração natural é um indicador direto da evolução de um sistema implantado rumo à pretendida diversidade e sustentabilidade.

Partindo da hipótese de que plantios de leguminosas arbóreas fixadoras de $\mathrm{N}_{2}$ podem funcionar como condicionadores do substrato e facilitadores da sucessão natural, estudou-se a composição florística e fitossociológica da regeneração natural de uma encosta reflorestada com leguminosas arbóreas em 1993, em Angra dos Reis, RJ.

R. Árvore, Viçosa-MG, v.28, n.6, p.801-809, 2004

\section{MATERIAL E MÉTODOS}

\subsection{Caracterização da Área}

A área em estudo situa-se no Condomínio Portogalo, município de Angra dos Reis, RJ, (2302'30'S; 44¹1'30'W; altitude em torno de $150 \mathrm{~m}$ ) e está inserida no domínio da Floresta Ombrófila Densa (VELOSO et al., 1991). O clima da região, segundo Köppen, caracterizase como Af - tropical úmido, com precipitações anuais da ordem de $2.300 \mathrm{~mm}$, sem estação seca definida. A encosta estudada - com cerca de 3 ha - foi reflorestada em 1993, no espaçamento de $2 \mathrm{~m}$ x 3 m, com uma mistura de espécies de leguminosas florestais, em que Acacia mangium Willd., Acacia auriculiformis A. Cum. ex Benth. e Mimosa tenuiflora (Willd.) Poiret correspondem $50 \%$ das mudas. Hoje, as três espécies representam cerca de $90 \%$ dos indivíduos plantados.

\subsection{Coleta, manuseio e identificação do material botânico}

O material botânico foi coletado em setembro e outubro de 2000. A identificação das exsicatas foi realizada através de bibliografia especializada, da comparação com material depositado no Herbário RBR do Dept. de Botânica da UFRRJ, através do auxílio de especialistas. O material botânico coletado ficou depositado na mesma instituição. A nomenclatura das famílias botânicas foi feita com base na proposição de Judd et al. (1999).

\subsection{Levantamentos florístico e fitossociológico e análise de DADOS}

A encosta reflorestada foi dividida em três terços - superior, declividade média de $60 \%$ (Reflo 1), médio, declividade média de $45 \%$ (Reflo 2 ) e inferior, declividade média de $10 \%$ (Reflo 3). Em cada terço foi delimitada uma parcela de $800 \mathrm{~m}^{2}(20 \mathrm{~m}$ x $40 \mathrm{~m})$, subdividida em quatro subparcelas de $200 \mathrm{~m}^{2}(10 \mathrm{~m}$ x $20 \mathrm{~m})$. Abaixo da encosta reflorestada, a cerca de $200 \mathrm{~m}$ situa-se um fragmento florestal (1,5 a 2,0 ha), identificado como a fonte mais próxima de propágulos exógenos para a área do reflorestamento e onde foi marcada mais uma parcela, também de $800 \mathrm{~m}^{2}$ (Mata Secundária).

Nas subparcelas, realizou-se o levantamento florístico e fitossociológico da regeneração, amostrando todos os indivíduos a partir de $40 \mathrm{~cm}$ de altura, os quais receberam etiquetas de plástico numeradas seqüencialmente, afixadas com fio plástico. No fragmento de Mata Secundária foram amostrados todos os 
indivíduos com DAP superior ou igual a $5 \mathrm{~cm}$, também recebendo etiquetas de plástico numeradas seqüencialmente e afixadas com prego. Para o cálculo dos parâmetros fitossociológicos foi utilizado o programa FITOPAC, versão 2.0, desenvolvido pelo Prof. Dr. George J. Shepherd, da Universidade Estadual de Campinas. A análise foi realizada de cada área dentro do reflorestamento (diferentes declividades), para as três parcelas em conjunto e na área de Mata Secundária, empregando-se as fórmulas apresentadas por Mueller-
Dombois e Ellenberg (1974) para freqüência, densidade e dominância, valor de importância (VI) e valor de cobertura (VC). Foram utilizados também os índices de diversidade de Shannon e o índice de uniformidade de Pielou (1975).

\section{RESULTADOS E DISCUSSÃO}

Foram amostradas 50 espécies na regeneração natural pertencentes a 25 famílias botânicas, sendo 24 em Reflo 1, 32 em Reflo 2 e também 32 em Reflo 3 (Tabela 1).

Tabela 1 - Lista das espécies de regeneração natural amostradas em uma encosta reflorestada com leguminosas arbóreas, em Angra dos Reis, RJ

Table 1 - List of native species regeneration under a legume tree stand on a hillside at Angra dos Reis, RJ

\begin{tabular}{|c|c|c|c|c|}
\hline Famílias & Espécies & Reflo 1 & Reflo 2 & Reflo 3 \\
\hline Anacardiaceae & Schinus terebinthifolius $\operatorname{Radd} i$ & & & $\mathrm{X}$ \\
\hline Combretaceae & Terminalia $\mathrm{sp}$. & & & $\mathrm{X}$ \\
\hline \multirow[t]{3}{*}{ Compositae } & Compositae 1 & $\mathrm{X}$ & $\mathrm{X}$ & $\mathrm{X}$ \\
\hline & Compositae 2 & & & $X$ \\
\hline & Vernonia sp. & & $\mathrm{X}$ & \\
\hline Erythroxylaceae & Erythroxyllum pulchrum A St. Hil. & $\mathrm{X}$ & $\mathrm{X}$ & $\mathrm{X}$ \\
\hline \multirow[t]{3}{*}{ Euphorbiaceae } & Alchornea glandulosa Poepp. \& Endl. & $\mathrm{X}$ & $\mathrm{X}$ & $\mathrm{X}$ \\
\hline & Euphorbiaceae 1 & & $X$ & \\
\hline & Pera af. glabrata (Schott) Baill & $\mathrm{X}$ & & \\
\hline Flacourtiaceae & Casearia af. sylvestris $\mathrm{Sw}$. & & $\mathrm{X}$ & $\mathrm{X}$ \\
\hline Gramineae & Bambusa sp. & $\mathrm{X}$ & $\mathrm{X}$ & $\mathrm{X}$ \\
\hline Lauraceae & Nectandra membranacea (Sw.) Griseb. & $\mathrm{X}$ & $\mathrm{X}$ & $X$ \\
\hline Leguminosae & Pterocarpus rohrii Vahl & & & $X$ \\
\hline \multirow[t]{5}{*}{ Melastomataceae } & Melastomataceae 1 & $\mathrm{X}$ & & \\
\hline & Melastomataceae 2 & & $\mathrm{X}$ & \\
\hline & Miconia sp1 & & & $\mathrm{X}$ \\
\hline & Miconia sp2 & $\mathrm{X}$ & $\mathrm{X}$ & $\mathrm{X}$ \\
\hline & Tibouchina granulosa Cogn. & $\mathrm{X}$ & & \\
\hline \multirow[t]{2}{*}{ Meliaceae } & Guarea guidonia (L.) Sleumer & $\mathrm{X}$ & $\mathrm{X}$ & $\mathrm{X}$ \\
\hline & Trichilia af. lepidota Martius & & $\mathrm{X}$ & $\mathrm{X}$ \\
\hline Monimiaceae & Siparuna guianensis Aubl. & $\mathrm{X}$ & $\mathrm{X}$ & \\
\hline \multirow[t]{3}{*}{ Moraceae } & Cecropia $\mathrm{sp}$ & $\mathrm{X}$ & $X$ & $\mathrm{X}$ \\
\hline & Ficus insipida Willd. & $X$ & & \\
\hline & Moraceae 1 & & & $\mathrm{X}$ \\
\hline \multirow[t]{2}{*}{ Musaceae } & Heliconia psitacorum $\mathrm{L}$. & & $\mathrm{X}$ & $\mathrm{X}$ \\
\hline & Heliconia $\mathrm{sp} 2$ & & & $\mathrm{X}$ \\
\hline Myrsinaceae & Rapanea ferruginea (Ruiz et Pav) Mez & $\mathrm{X}$ & $\mathrm{X}$ & $\mathrm{X}$ \\
\hline \multirow[t]{5}{*}{ Myrtaceae } & Campomanesia sp. & $\mathrm{X}$ & $\mathrm{X}$ & \\
\hline & Calyptranthes sp. & & $\mathrm{X}$ & \\
\hline & Eugenia uniflora $\mathrm{L}$. & & $\mathrm{X}$ & $\mathrm{X}$ \\
\hline & Myrcia sp1 & & & $\mathrm{X}$ \\
\hline & Myrtaceae 1 & & & $\mathrm{X}$ \\
\hline \multirow[t]{3}{*}{ Piperaceae } & Piper arboreum Aubl. var. arboreum & & $\mathrm{X}$ & $\mathrm{X}$ \\
\hline & Piper mollicomum Kunth & $\mathrm{X}$ & $\mathrm{X}$ & $\mathrm{X}$ \\
\hline & Pothomorphe umbellata (L.) Miq. & $\mathrm{X}$ & $\mathrm{X}$ & \\
\hline
\end{tabular}


Quadro 1 - cont.

Table 1 - cont.

\begin{tabular}{|c|c|c|c|c|}
\hline Famílias & Espécies & Reflo 1 & Reflo 2 & Reflo 3 \\
\hline Polygonaceae & Triplaris sp. & & & $\mathrm{X}$ \\
\hline Rubiaceae & Psychotria velloziana Benth. & $\mathrm{X}$ & & $\mathrm{X}$ \\
\hline Sapindaceae & Cupania oblongifolia Mart. & & & $\mathrm{X}$ \\
\hline \multirow[t]{7}{*}{ Solanaceae } & Solanaceae 1 & & $\mathrm{X}$ & \\
\hline & Solanaceae 2 & & $\mathrm{X}$ & \\
\hline & Solanaceae 3 & $\mathrm{X}$ & & \\
\hline & Solanaceae 4 & $\mathrm{X}$ & $\mathrm{X}$ & $\mathrm{X}$ \\
\hline & Solanaceae 5 & $\mathrm{X}$ & $\mathrm{X}$ & \\
\hline & Solanaceae 6 & & & $\mathrm{X}$ \\
\hline & Solanum argenteum Dun & & $\mathrm{X}$ & \\
\hline Ulmaceae & Trema micrantha (L.) Blume & & $\mathrm{X}$ & $\mathrm{X}$ \\
\hline Urticaceae & Urticaceae 1 & $\mathrm{X}$ & $\mathrm{X}$ & $\mathrm{X}$ \\
\hline \multirow[t]{2}{*}{ Verbenaceae } & Aegyphilla af. sellowiana Cham. & $\mathrm{X}$ & $\mathrm{X}$ & $\mathrm{X}$ \\
\hline & Lantana camara $\mathrm{L}$. & $\mathrm{X}$ & $\mathrm{X}$ & \\
\hline Indet & Indet 1 & & $\mathrm{X}$ & \\
\hline
\end{tabular}

O critério de inclusão adotado e o próprio estágio inicial de regeneração em que a área se encontra dificultaram tanto a identificação do material coletado quanto, muitas vezes, a própria coleta; a fim de conseguir material suficiente para a herborização, danificava-se muito o indivíduo.

Na comunidade como um todo, as famílias com maior número de indivíduos foram: Meliaceae (298), Euphorbiaceae (70), Piperaceae (64), Lauraceae (41), Gramineae (40) e Moraceae (37), respondendo por quase $80 \%$ dos indivíduos amostrados (Figura 1). Quanto ao número de espécies por família, as de maior representatividade foram Solanaceae (7), Melastomataceae (5) e Myrtaceae (5) (Figura 2).

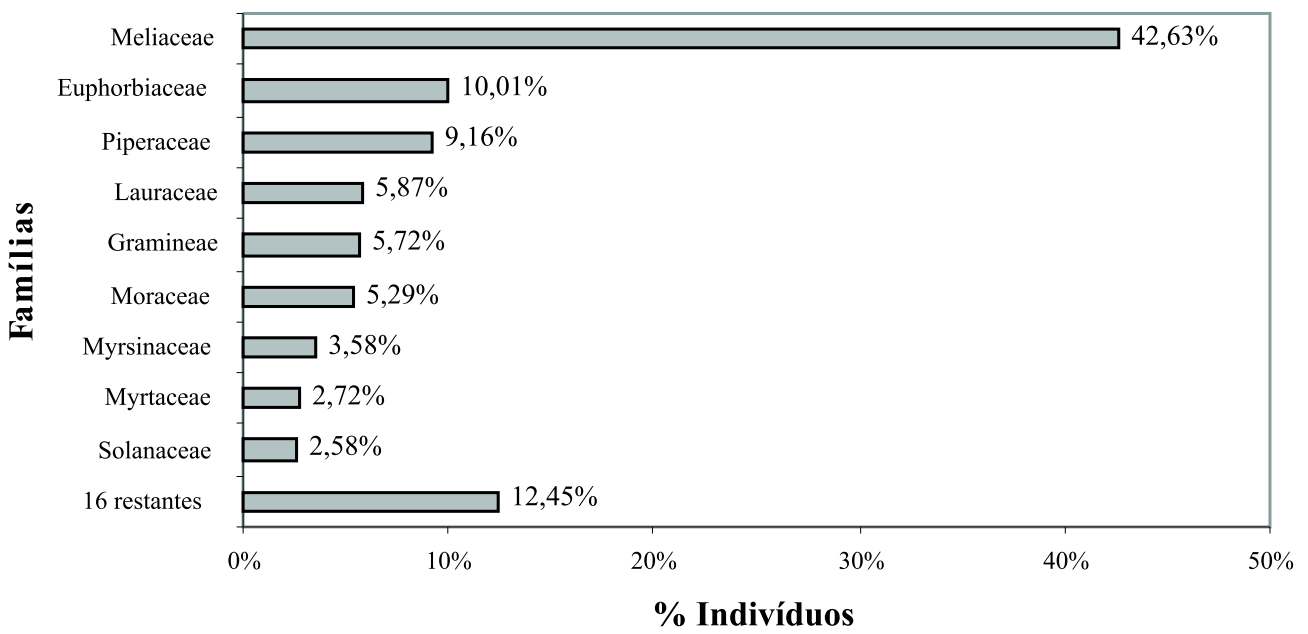

Figura 1 - Distribuição do número de indivíduos por família, em porcentagem do número total de indivíduos amostrados nas três áreas do reflorestamento com leguminosas arbóreas, em Angra dos Reis, RJ.

Figure 1 - Percent distribution of individuals per family found under a legume tree stand on a hillside at Angra dos Reis, RJ.

R. Árvore, Viçosa-MG, v.28, n.6, p.801-809, 2004 


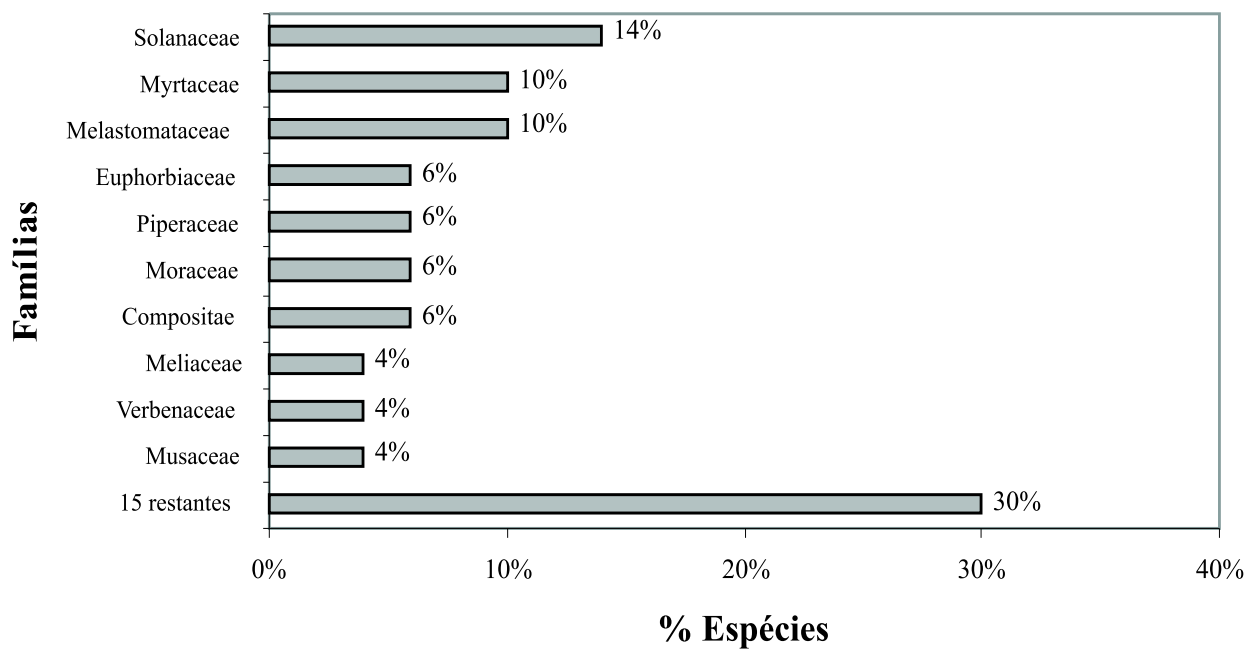

Figura 2 - Distribuição do número de espécies por família, em porcentagem do número total de espécies amostradas nas três áreas do reflorestamento com leguminosas arbóreas, em Angra dos Reis, RJ.

Figure 2 -Percent distribution of species per family found under a legume tree stand on a hillside at Angra dos Reis, RJ.

Oliveira (1999), em uma área de roçado abandonada há 5 anos, na Ilha Grande, amostrou 26 espécies distribuídas em 17 famílias (critério de inclusão: altura maior que $2 \mathrm{~m}$ e Perímetro à Altura do Peito (PAP) maior que $8 \mathrm{~cm}$ ), em que se destacaram com maior riqueza de espécies as famílias Leguminosae e Melastomataceae (15,4\% cada), seguidas de Solanaceae $(11,4 \%)$. Apesar do diferente critério de inclusão, as duas áreas apresentaram duas famílias de maior riqueza de espécies em comum - Melastomataceae e Solanaceae - típicas de estágios iniciais de sucessão. Chama a atenção na encosta reflorestada com leguminosas pioneiras a ausência dessa mesma família na regeneração natural. Digna de nota é, também, a ausência absoluta das espécies plantadas na regeneração, afastando a hipótese levantada por alguns autores (REIS et al., 1996) de que as espécies plantadas possam vir a tornar-se pragas e prejudicar o desenvolvimento da sucessão. Tal situação não tem ocorrido em plantios de leucena, por exemplo. Freire et al. (2000) verificaram que $98 \%$ dos indivíduos de regeneração natural sob plantios de Leucaena leucocephala (Lam.) deWit. foram dessa mesma espécie. Em cada uma das situações, entretanto, precisam ser considerados outros fatores, entre os quais se destaca a existência de remanescentes florestais próximos e com boa diversidade de espécies que funcionem como dispersores de propágulos.

$\mathrm{Na}$ área de Mata Secundária foram amostradas 16 espécies pertencentes a 13 famílias referentes a indivíduos com DAP superior ou igual a $5 \mathrm{~cm}$ (Tabela 2), sendo oito dessas espécies também encontradas na área do reflorestamento.

Apesar da pequena área amostral $\left(800 \mathrm{~m}^{2}\right)$ e do pequeno número de famílias e espécies amostradas, a curva espécie x área (Figura 3) exibiu suficiência amostral, evidenciando a baixa diversidade florística do fragmento, confirmando tratar-se de área de vegetação secundária, caracterizada por baixa diversidade de espécies (MANTOVANI, 1993).

No fragmento de Mata Secundária estudado, as famílias com maior número de indivíduos foram: Meliaceae (20), Nyctaginaceae (10), Lauraceae (5) e Moraceae e Sapindaceae, com três indivíduos cada uma, respondendo estas cinco famílias por quase $80 \%$ dos indivíduos amostrados (Figura 4). Das 13 famílias encontradas, 10 estão representadas por uma única espécie e as três restantes, por duas espécies.

R. Árvore, Viçosa-MG, v.28, n.6, p.801-809, 2004 
Tabela 2 - Lista das espécies arbóreas com DAP superior ou igual a $5 \mathrm{~cm}$, amostradas em fragmento de mata secundária, em Angra dos Reis, RJ

Table 2 - List of tree species with DBH equal or above $5 \mathrm{~cm}$ sampled in a secondary forest fragment at Angra dos Reis, $R J$

\begin{tabular}{ll}
\hline Famílias & \multicolumn{1}{c}{ Espécies } \\
\hline Apocynaceae & Peschieria af. laeta Mart ex. A. DC \\
Arecaceae & Euterpe edulis Mart. \\
Boraginaceae & Cordia magnoliefolia Cham. \\
Erythroxylaceae & Erythroxylum pulchrum A.St. Hil. \\
Flacourtiaceae & Casearia sp. \\
Lauraceae & Nectandra membranacea (Swart) Griseb \\
Leguminosae Mimosoideae & Inga sp. \\
Meliaceae & Guarea guidonia (L.) Sleumer \\
Monimiaceae & Meliaceae 1 \\
Moraceae & Siparuna guianensis Aubl. \\
Myrtaceae & Cecropia sp. \\
Nyctaginaceae & Ficus insipida Willd. \\
Sapindaceae & Myrtaceae 2 \\
\hline
\end{tabular}

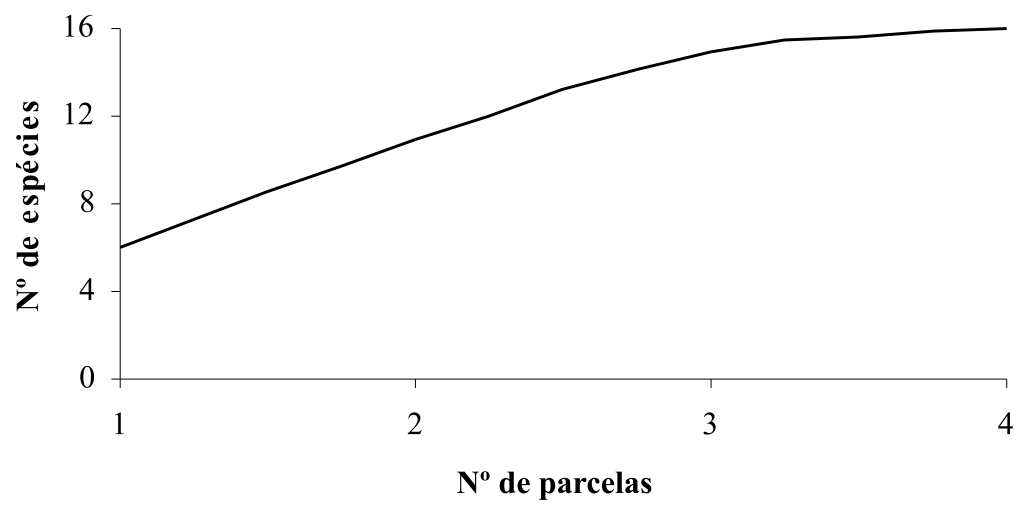

Figura 3 - Curva do número de espécies pelo número de parcelas amostrais em fragmento de mata secundária, em Angra dos Reis, RJ.

Figure 3 - Species area curve sampled in a Secondary Forest fragment at Angra dos Reis, RJ.

Nas três áreas do reflorestamento e também no fragmento de Mata Secundária, Guarea guidonia, a carrapeta foi a espécie de maior valor de importância (VI), com o maior número de indivíduos e com os valores mais elevados tanto para densidade quanto para dominância relativas. Para Reflo 3 e para o fragmento de Mata Secundária só esta espécie representou mais de 1/3 do VI. A espécie com o segundo maior índice variou nas quatro áreas: em Reflo 1 foi a Bambusa sp.
(36,02\%), em Reflo 2 a Cecropia sp. (30,04\%), em Reflo 3 a Alchornea glandulosa $(28,94 \%)$ e no fragmento de Mata Secundária foi a Guapira sp. (30,03\%), espécie não ocorrente na regeneração natural da área reflorestada. Das oito espécies do fragmento florestal que ocorrem na regeneração do reflorestamento, quatro ocorrem nas três áreas: Guarea guidonia, Nectandra membranacea, Cecropia sp.e Erythroxylum pulchrum. Essas quatro espécies representam 53,4\% do VI na

R. Árvore, Viçosa-MG, v.28, n.6, p.801-809, 2004 
Mata Secundária 50,5\% do VI em Reflo 3, 41,9\% em Reflo 2 e $34,7 \%$ em Reflo 1.

A Tabela 3 sintetiza uma série de informações sobre as áreas estudadas. A grande densidade de indivíduos de Guarea guidonia diminuiu a eqüitabilidade da regeneração na área reflorestada, refletindo também no Índice de Shannon mais baixo para Reflo 3, apesar da maior densidade e riqueza observada nessa área.

O Índice de Shannon observado no fragmento de Mata Secundária estudado está de acordo com valores encontrados em outros estudos de diversidade de fragmentos florestais. Piña-Rodrigues et al. (1999) encontraram o valor de 1,64 e 1,04 em fragmentos de baixa diversidade e menores que 2 ha e 2,34 em um fragmento entre 3 e 5 ha, na região de Paty do Alferes, no Rio de Janeiro.

Como a regeneração do reflorestamento e o fragmento de Mata Secundária foram amostrados segundo critérios de inclusão distintos, não se pode fazer comparações entre os índices de diversidade ou equabilidade calculados para as duas áreas.

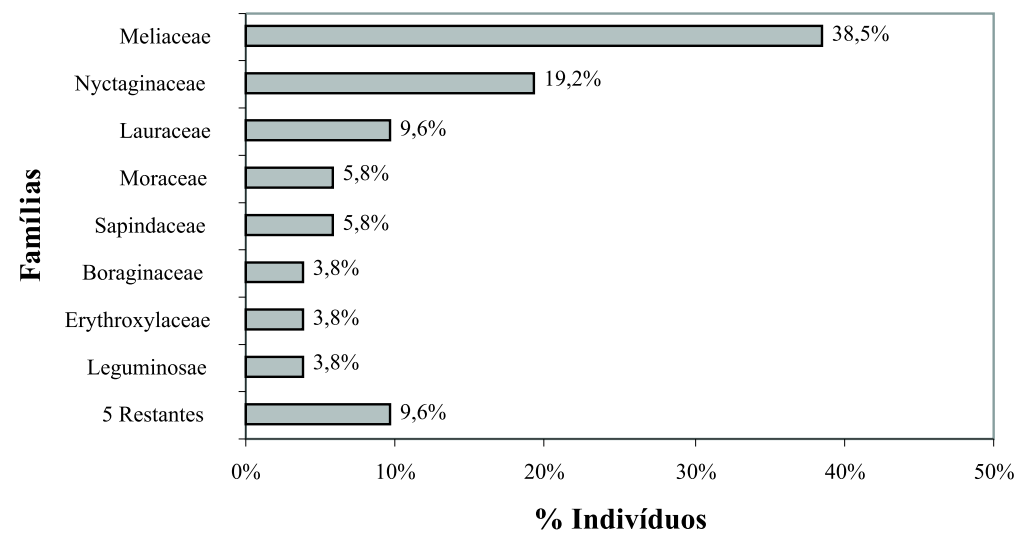

Figura 4 - Distribuição do número de indivíduos por família, em porcentagem do número total de indivíduos amostrados em fragmento de mata secundária, em Angra dos Reis, RJ.

Figure 4 - Percent distribution of individuals family ${ }^{-1}$ in a Secondary forest fragment at Angra dos Reis, RJ.

Tabela 3 - Síntese das informações obtidas sobre a sucessão secundária em uma encosta reflorestada com leguminosas pioneiras e sobre um fragmento de mata secundária no Condomínio Portogalo, em Angra dos Reis, RJ

Table 3 - Phytosociological parameters obtained in natural regeneration stands under legume tree stands and a Secondary Forest fragment at Angra dos Reis, RJ

\begin{tabular}{|c|c|c|c|c|c|}
\hline Parâmetros & Reflo 1 & Reflo 2 & Reflo 3 & Reflo 123 & Mata \\
\hline Área amostrada $(\mathrm{m} 2)$ & 800 & 800 & 800 & 2.400 & 800 \\
\hline $\mathrm{N}^{\circ}$ de indivíduos & 125 & 223 & 351 & 699 & 52 \\
\hline $\mathrm{N}^{\circ}$ de famílias & 16 & 19 & 22 & 25 & 13 \\
\hline $\mathrm{N}^{\mathrm{o}}$ de espécies & 24 & 32 & 32 & 50 & 16 \\
\hline Índice de Shannon & 2,59 & 2,53 & 1,90 & 2,38 & 2,17 \\
\hline Densidade total (ind/ha) & $1.562,5$ & $2.787,5$ & $4.387,5$ & $2.912,5$ & 650 \\
\hline Altura máxima $(\mathrm{m})$ & 3,47 & 5,08 & 4,9 & 5,08 & 25,00 \\
\hline Altura média (m) & 1,10 & 1,29 & 1,43 & 1,32 & 10,89 \\
\hline Diâmetro máximo (cm) & $3,28 *$ & $4,70 *$ & $4,60 *$ & $4,70 *$ & $97,00 * *$ \\
\hline Diâmetro médio $(\mathrm{cm})$ & $1,25 *$ & $1,31 *$ & $1,68 *$ & $1,49 *$ & $20,00 * *$ \\
\hline
\end{tabular}

**DAP - diâmetro à altura do peito.

* DAC - diâmetro à altura do colo. 


\section{CONCLUSÕES E CONSIDERAÇÕES FINAIS}

Diante do exposto, chegou-se às seguintes conclusões:

- O reflorestamento com leguminosas arbóreas mostrou-se eficaz na ativação dos mecanismos de sucessão natural e, após 7 anos, 50 espécies de 25 famílias botânicas já colonizam o sub-bosque dessa área, com a ressalva de que algumas espécies plantadas já começam a entrar em senescência, com a tendência de saírem paulatinamente do sistema.

- A evolução da sucessão natural apresentou um gradiente de desenvolvimento em função da menor declividade e menor distância dos remanescentes florestais, com maior densidade de indivíduos e maior riqueza de espécies na área de menor declividade e mais próxima do fragmento florestal.

- Guarea guidonia foi a espécie de maior Valor de Importância (VI), tanto para o reflorestamento quanto para o fragmento de Mata Secundária estudado.

- Apesar da concordância entre as espécies de maior VI no reflorestamento e no fragmento de Mata Secundária estudado, a riqueza e diversidade de espécies encontradas na regeneração natural indicaram que o reflorestamento recebe propágulos provenientes também de outros fragmentos, de distâncias maiores.

\section{REFERÊNCIAS BIBLIOGRÁFICAS}

CALEGÁrio, N. Parâmetros florísticos e fitossociológicos de regeneração natural de espécies arbóreas nativas no sub-bosque de povoamento de Eucalyptus, no município de Belo Oriente, MG. 1993. 114 f. Dissertação (Mestrado em Ciência Florestal). Universidade Federal de Viçosa, Viçosa, 1993.

CAMPELlO, E. F.C. A Influência de leguminosas arbóreas fixadoras de nitrogênio na sucessão vegetal em áreas degradadas na Amazônia.1999. 121 f. Tese (Doutorado em Ciência Florestal) Universidade Federal de Viçosa, Viçosa, 1999.

FRANCO, A. A. et al. Revegetação de solos degradados. Seropédica: Embrapa-CNPBS, 1992. 8 p. (Embrapa-CNPBS. Comunicado Técnico, 9).

R. Árvore, Viçosa-MG, v.28, n.6, p.801-809, 2004
FRANCO, A. A. et al. Uso de leguminosas florestais noduladas e micorrizadas como agentes de recuperação e manutenção da vida no solo: um modelo tecnológico. Oecologia Brasiliensis, v.1, p.459-467, 1995.

FRANCO, A. A.; FARIA, S. M. The contribution of $\mathrm{N}_{2}$-fixing tree legumes to land reclamation and sustainability in the tropics. Soil Biology Biochemistry, v. 29, n. 5/6, p. 897-903, 1997.

FREIRE, J.M. et. al. Regeneração natural sob plantios com predominância de Leucaena leucocephala (Lam) de Wit., Mimosa caesalpiniaefolia Benth. e plantios com maior diversidade de espécies em madureira, RJ. In: FOREST, Porto Seguro, 2000. Anais... Porto Seguro, 2000.

HAGGAR, J.; WIGHTMAN, K.; FISHER, R. The potential of plantations to foster woody regeneration within a deforested landscape in lowland Costa Rica. Forest Ecology and Management, v.99, p.55-64, 1997.

JUDD, W.S.; CAMPBELL, C.S.; KELLOGG, E.A; STEVENS, P.F. Plant systematics: a phylogenetic approach. Suderland: Sinner Associates, 1999. 464 p.

KAGEYAMA, P. et al. Revegetação de Áreas Degradadas: Modelos de Consorciação com Alta Diversidade. In: SIMPÓSIO SUL AMERICANO, 1.; SIMPÓSIO NACIONAL DE RECUPERAÇÃO DE ÁREAS DEGRADADAS, 2.; 1994, Foz do Iguaçu Anais.... Curitiba: FUPEF, 1994. v. 2, p 569-576.

MANTOVANI, W. Estrutura e dinâmica da floresta atlântica em Iguape, SP. 1993. 126 p. Tese (Livre-Docência) - Universidade de São Paulo, São Paulo, 1993.

MUELLER-DOMBOIS, D.; ELLENBERG, H. Aims and methods of vegetation ecology. New York: John Wiley \& Sons, 1974. 547 p. 
OLIVEIRA, R. R. O rastro do homem na floresta: sustentabilidade e funcionalidade da Mata Atlântica sob manejo caiçara. 1999. 148 f. Tese (Doutorado em Geografia) - Universidade Federal do Rio de Janeiro, Rio de Janeiro, 1999.

PARROTA, J. A. Secondary forest regeneration on degraded tropical lands: the role of plantations as “foster ecosystems". In: LEITE, H. e LOHMANN, M. (Eds), Restoration of Tropical Forest Ecosystems. Dordrecht: Kluwer, 1993. p.63-73.

PIELOU, E.C. Ecological diversity. New York: John Wiley \& sons, 1975. 165 p.

PIÑA-RODRIGUES, F.C.M.; COSTA, L.G.S.; REIS, A. Estratégias de estabelecimento de espécies arbóreas e o manejo de florestas tropicais. In: CONGRESSO FLORESTAL BRASILEIRO, 6., 1990, Campos do Jordão, SP. Anais... São Paulo: 1990. p.676-684.
REIS, A.; NAKAZONO, E.M.; MATOS, J. Z. Utilização da sucessão e das interações planta-animal na recuperação de áreas florestais degradadas. In: Recuperação de Áreas Degradadas, 3. Curso de Atualização. Curitiba: 1996, p. 29-43.

SILVA JÚNIOR, M. C.; SCARANO, F. R.; CARDEL, F. S. Regeneration of an Atlantic forest formation in the understorey of a Eucaliptus grandis plantation in southeastern Brazil. Journal of Tropical Ecology, 11: 147-152, 1995.

VELOSO, H.P.; RANGEL-FILHO, A. L. R.; LIMA, J. C. A. Classificação da vegetação brasileira adaptada a um sistema universal. Rio de Janeiro: IBGE, 1991. p. 124. 